\title{
Disparities between industrial and surgical ergonomics
}

\author{
F. Jacob Seagull \\ Department of Medical Education, G1211 Towsley Center, 1500 E. Medical Center Drive, SPC-5201, University \\ of Michigan Medical School, Ann Arbor, MI 48109 USA. jseagull@umich.edu
}

\begin{abstract}
A surgeon's work environment and working conditions are often harsher than those of an industrial worker. Accepted principles and regulations of ergonomics in manufacturing are largely ignored or absent in the medical/surgical domain. Examples include poor surgical tool handle design, awkward and stressful surgical postures, and prolonged standing without breaks and without a foot mat. In these and other areas, there are documented "best practices" for industrial hygiene and ergonomics that are not yet widely accepted for surgery. There is support in the literature for innovations in surgical ergonomics, yet adoption is not widespread. In the absence of these ergonomic principles, surgical repetitive strain injuries in minimally invasive surgery are reaching epidemic levels. As ergonomists, it falls upon us to understand why current solutions have not been widely adopted within this domain, and to derive solutions to the unique challenges of surgery.
\end{abstract}

Keywords: Industrial Ergonomics, Operating Room, Surgery, Standards, Tool Design

\section{Introduction}

Medical technology is exhibiting growth and development at a pace unrivaled in any previous time in history. Modern medicine provides sophisticated lifesaving technologies such as artificial hearts, surgical robotics, advanced imaging, informatics, and computer-guided surgical navigation systems [20]. New devices are continually being introduced to the operating room. These advanced technologies have reduced morbidity and mortality for patients, and brought about interventions to improve the quality of patients' lives. Yet despite all these advances, the room in which surgery takes place remains fundamentally unchanged for the surgeon. While technology advances, the human element that wields the scalpel is still subjected to a harsh working environment.

This harsh working environment can impact the safety of the patient and the safety of the surgeon. The stressful environment can lead to fatigue and pain, which may limit surgeons' margin of safety, and possibly contribute to poorer outcomes. The principles of ergonomic design of the workspace, task and tools may hold some value in mitigating the stress and improving performance.

\section{State of surgical ergonomics}

Ergonomic principles can be applied to a range of aspects of the surgical environment. The quality of the environment is not consistent across these different aspects.

Some aspects of surgical ergonomics are near optimal. Lighting in the operating room is recognized as essential to task performance, and is a key aspect of an operating room. Lighting of the surgical field typically includes multiple light sources that are high intensity, with adjustable brightness and flexible location. Similarly, environmental temperature and air quality generally are well regulated and pose no threat to the health and wellbeing of the surgeon.

Other aspects of surgical ergonomics, such as surgical instrument design, present a conundrum. On one hand, the array of instruments available reflects an excessive attention to optimizing instruments to specialized functions. Some of the earliest ergonomic studies involved optimizing tools for particular tasks, such as the seminal work by Taylor optimizing shovels for coal workers [21]. In the domain of surgical tools, for example, there are at least 162 classes of 
surgical instruments used in general surgery alone [9]. Tools are often adapted by surgeons continually to fit the specific needs of a given procedure or situation. In contrast to the wide array of instruments, there are very few variations in the grip of the instruments, and seemingly little work has been done with optimizing the handles. The vast majority of the customization takes place on the "sharp" end of the instrument.

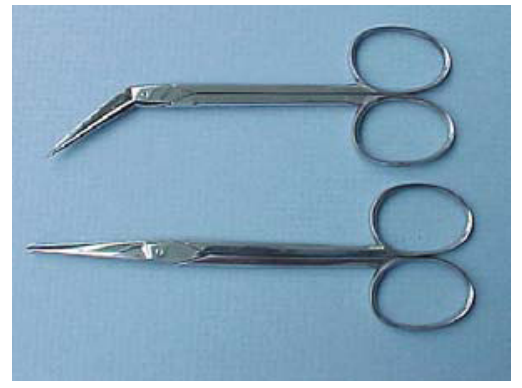

Fig. 1. Surgical scissors from a civil-war era surgical kit, circa 1850 [Collection of Dr. Michael Echols, used by permission].

Not only is the design of handles comparatively limited, but also aspects of some of the most commonly used designs used have remained the same for over a century. For example, a typical pair of surgical scissors has simple loop handles (Figure 1). These handles are typically thin, nearly circular, and symmetrical. In contrast, scissors used outside the operating room vary widely in design, but often have molded handles for distributing pressure and avoiding injury. They are commonly available in right and left handed versions, as well as a range of sizes (child, adult, etc.) to accommodate different hand sizes (Figure 2).

Surgical gloves are made to fit hands of varying sizes, and gloves typically range from size 5.5 to size 9. A given instrument that fits into those hands is typically available only in a single size [1]. A mismatch between the hand and instrument can have significant negative consequences. These mismatches are more evident in certain domains of surgery. Minimally Invasive Surgery (MIS), for example, faces particularly difficult challenges. In minimally invasive surgery, procedures have been designed to facilitate minimal damage to the patient's tissue. Unfortunately, the convenience to the patient comes at the price of a number of additional constraints on the surgeons. These constraints have resulted in ergonomic challenges to MIS practitioners.

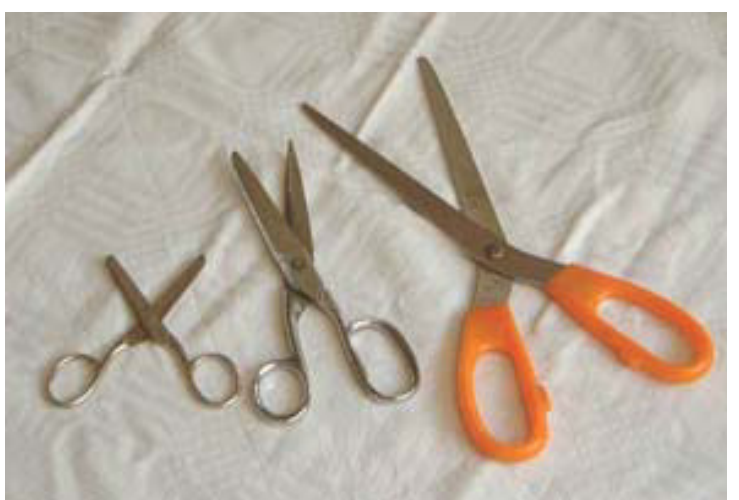

Fig. 2. Scissors with varying handle shape, size and material. [photo by Tarquin, used by permission].

Laparoscopic surgeons who use smaller gloves (i.e. have smaller hands) are significantly more likely to report musculoskeletal problems [1]. Those outcomes are not rare, and the prevalence of musculoskeletal complaints in laparoscopic surgeons is not limited to hands. Over $86 \%$ of laparoscopic surgeons [14] and $94 \%$ of surgeons performing Mohs surgery [7] reported physical symptoms associated with surgery, with complaints ranging from their hands arms and lower extremities to their eyes, neck and back [7, 14].

There are some other aspects of ergonomics in surgery that are unequivocally poor. Surgeons endure long durations of work in static or near static standing postures on hard surfaces with few if any breaks [15]. The displays of patient monitors are often placed in awkward positions, resulting in awkward neck postures $[15,23]$. Workplace layout is often sub-optimal $[19,25]$.

\section{Surgery as a Unique Domain}

There are constraints to improving the environment in every workplace. The surgical work environment is burdened with a number of constraints and barriers to improving ergonomics. These include the organic nature of the workspace, uniqueness of each patient, the environmental and regulatory demands, and the time sensitive nature of the work.

- Organic workspace: The anatomy of a human patient is not subject to redesign for optimal work environment. The geometry of the human body (patient) is an immutable constraint on the 
positioning of the surgeon and surgical tools. Optimizing is therefore difficult.

- Unique patients: Because people are not all identical, standard work is difficult to establish. Anatomical variation between patients necessitates variations in procedure, variations in tool placement and variations in tool use.

- Time-sensitive work: Surgical procedures often last for hours. This despite the fact that surgeons strive to minimize procedure length for a number of reasons. Operating room time is an expensive commodity. Additionally, shorter procedure duration is associated with faster recovery and better outcomes [e.g. 4, 16]. This time pressure may be a factor in the dearth of regularly scheduled breaks for surgeons. Surgeons also may be reluctant to take a break in the middle of a procedure for reasons associated with maintaining continuity of care.

- Environmental and regulatory demands: Surgical instruments are subject to strict regulatory requirements. In America, the Food and Drug Administration (FDA) regulates instruments as medical devices. Instruments that are nondisposable must be constructed so that they can be safely cleaned. This restricts both the materials that can be used (e.g. usually non-porous) and the geometry of the instrument (e.g. to allow effective cleaning without reservoirs for contaminants). These rigorous demands constrain optimal ergonomic designs.

All of these aspects of the surgical domain create an environment in which common industrial ergonomic solutions may not be applicable.

\section{Long-Standing Problems}

Ergonomists have been decrying the lack of sound ergonomic practices in the operating room since at least 1914 [5]. Since that time, the field of industrial ergonomics has amassed a significant body of evidence supporting some basic principles of good ergonomic work design. There is consensus regarding a number of risk factors that should be avoided in the work environment in order to minimize risk of injury. Despite these constraints, there may be simple principles that can help minimize these risks. These risk factors include use of force, repetition, awkward postures, static postures, and exposure to vibration [2, 13].
For example, remedies for prolonged standing work have been implemented to some extent in industry. The use of anti-fatigue floor matting is mandated by some labor unions in the US for workers who stand for prolonged periods, and the US Occupational Health and Safety Administration (OSHA) recommends anti-fatigue matting for those a number of specific job-types that involve prolonged standing $[6,17]$. Lower extremity injury and has been studied extensively $[3,8,17]$. The use of anti-fatigue matting in the operating room is uncommon.

As mentioned previously, awkward postures and static postures are also known in industry as a source of musculoskeletal disorders. However, there is large variability in the way that surgeons position themselves with respect to the patient during surgery. Commonly used postures often mandate activity outside recommended safe ranges of motion, leading to increased risk of physical injury, even when there are better alternative postures that are well established [25].

Similarly, there have been significant levels of research in the surgical domain regarding ergonomics. Topics include the placement of patient monitors [11, $22,23]$, the height of the surgical table $[10,15,18]$, the contents of surgical equipment trays [19], instrument handle design $[12,18,24]$. Despite this research, poor environments still pervade operating rooms.

This conduct of controlled, empirical research into surgical ergonomics has proven necessary but not sufficient to correct the prevalent problems in the operating room. Education may be the missing link. There is a documented lack of awareness to ergonomic issues by surgeons. A majority (57\%) of Laparoscopic - arguably the surgeons most affected by MSDs - report little or no knowledge of ergonomics [14]. Resolving the problems of surgical ergonomics may require more than the development of the solutions themselves. Improving surgeon knowledge of sound, basic ergonomic principles and practices may be the most pressing agenda for surgical ergonomics.

\section{Conclusions}

Surgical ergonomics does not suffer from lack of attention. The foundations of industrial ergonomics were established nearly simultaneously with surgical ergonomics. Problems are well documented since at least 1911, and improved practice recommendations exist for a number of problematic practices in both 
industry and surgery $[5,21]$. While industrial ergonomics has flourished, penetration into the domain of surgery is only now improving, notably in Minimally Invasive Surgery, where the ergonomic problems are so pronounced. However, recommended practices and design-based solutions must take into account the unique nature of the surgical environments. A key to improving surgical ergonomics may lie not merely in research and design, but also in the relatively neglected domain of surgical education.

\section{References}

[1] Berguer R, Hreljac A. The relationship between hand size and difficulty using surgical instruments: a survey of 726 laparoscopic surgeons. Surg Endosc. 2004 Mar;18(3):508-12.

[2] Brouha L. 1973. Physiology in Industry. London: Pergamon Press.

[3] Cham R, Redfern MS. Effect of flooring on standing comfort and fatigue. Hum Factors. 2001 Fall;43(3):381-91

[4] Chu D, Bakaeen FG, Wang XL, Lemaire SA, Coselli JS, Huh $\mathrm{J}$. Does the duration of surgery affect outcomes in patients undergoing coronary artery bypass grafting? Am J Surg. 2008 Nov;196(5):652-6.

[5] Dickenson, RL. Standardization of Surgery: An Attack on the Problem. JAMA 1914: 63(9) 763-5.

[6] "Employer Payment for Personal Protective Equipment; Final Rule." Federal Register 72 (November 15, 2007 ). http://www.osha.gov/FedReg_osha_pdf/FED20071115.pdf (accessed on August 24, 2011).

[7] Esser AC, Koshy JG, Randle HW. Ergonomics in office-based surgery: a survey-guided observational study. Dermatol Surg. 2007 Nov;33(11):1304-13.

[8] Gell N, Werner RA, Hartigan A, Wiggermann N, Keyserling WM. Risk factors for lower extremity fatigue among assembly plant workers. Am J Ind Med. 2011 Mar;54(3):216-23.

[9] "Instruments used in General Surgery." Wikipedia. http://en.wikipedia.org/wiki/Instruments used in general sur gery (Retrieved August 25, 2011)

[10] Manasnayakorn S, Cuschieri A, Hanna GB. Ergonomic assessment of optimum operating table height for hand-assisted laparoscopic surgery. Surg Endosc. 2009 Apr;23(4):783-9.

[11] Matern U, Faist M, Kehl K, Giebmeyer C, Buess G. Monitor position in laparoscopic surgery. Surg Endosc. 2005 Mar;19(3):436-40. Epub 2005 Jan 10.

[12] Matern U, Kuttler G, Giebmeyer C, Waller P, Faist M. Ergonomic aspects of five different types of laparoscopic instru- ment handles under dynamic conditions with respect to specific laparoscopic tasks: an electromyographic-based study. Surg Endosc. 2004 Aug;18(8):1231-41

[13] National Academy of Sciences (1998). Work-Related Musculoskeletal Disorders: A Review of the Evidence. Washington, DC: National Academy Press.

[14]Park A, Lee G, Seagull FJ, Meenaghan N, Dexter D. Patients benefit while surgeons suffer: an impending epidemic. J Am Coll Surg. 2010 Mar;210(3):306-13.

[15]Pérez-Duarte FJ, Sánchez-Margallo FM, Díaz-Güemes Martín-Portugués I, Sánchez-Hurtado MA, Lucas-Hernández M, Usón Gargallo. Ergonomics in laparoscopic surgery and its importance in surgical training. J.Cir Esp. 2011 Jun 22.

[16] Procter LD, Davenport DL, Bernard AC, Zwischenberger JB. General surgical operative duration is associated with increased risk-adjusted infectious complication rates and length of hospital stay. J Am Coll Surg. 2010 Jan;210(1):60-5.e1-2.

[17] Redfern MS, Cham R. The influence of flooring on standing comfort and fatigue. AIHAJ. 2000 Sep-Oct;61(5):700-8.

[18] Sari V, Nieboer TE, Vierhout ME, Stegeman DF, Kluivers KB. The operation room as a hostile environment for surgeons: physical complaints during and after laparoscopy. Minim Invasive Ther Allied Technol. 2010 Apr;19(2):105-9.

[19] Seagull FJ, Mackenzie CF, Xiao Y, Bochicchio GV. Videobased ergonomic analysis to evaluate thoracostomy tube placement techniques. J Trauma. 2006 Jan;60(1):227-32.

[20] Seagull FJ, Moses GR, Park AE. Pillars of a Smart, Safe Operating Room. In: Henriksen K, Battles JB, Keyes MA, Grady ML, editors. Advances in Patient Safety: New Directions and Alternative Approaches (Vol. 3: Performance and Tools). Rockville (MD): Agency for Healthcare Research and Quality (US); 2008 Aug.

[21] Taylor FW. The Principles of Scientific Management (10th edition) 1911. [retrieved from www.ProjectGutenberg.org]

[22] Uhrich ML, Underwood RA, Standeven JW, Soper NJ, Engsberg JR.Assessment of fatigue, monitor placement, and surgical experience during simulated laparoscopic surgery. Surg Endosc. 2002 Apr;16(4):635-9.

[23] van Det MJ, Meijerink WJ, Hoff C, Totté ER, Pierie JP Optimal ergonomics for laparoscopic surgery in minimally invasive surgery suites: a review and guidelines. Surg Endosc. 2009 Jun;23(6):1279-85.

[24]van Veelen MA, Meijer DW, Uijttewaal I, Goossens RH, Snijders CJ, Kazemier G. Improvement of the laparoscopic needle holder based on new ergonomic guidelines. Surg Endosc. 2003 May; 17(5):699-703.

[25] Youssef Y, Lee G, Godinez C, Sutton E, Klein RV, George IM, Seagull FJ, Park A. Laparoscopic cholecystectomy poses physical injury risk to surgeons: analysis of hand technique and standing position. Surg Endosc. 2011 Jul;25(7):2168-74. 